\title{
Gradual Quadriparasis: a diagnostic dilemma
}

\author{
Dr. Piyudeo Mahant, Dr.Upasana Shobhane, Professor Seema Mahant, \\ Professor Rakesh Biswas \\ Associate Professor People's College of Medical Sciences, Radiodiagnosis HIG-B-10,PCMS campus,Bhanpur, \\ BhopalBhopal, M.P., IN 462037 \\ Assistant Professor People's College of Medical Sciences, Pathology People's College of Medical Sciences, \\ Bhanpur bypass road, Bhopal \\ Professor PCMS\&RC,Bhopal, Gen.Medicine HIG-B-10 PCMS campus Bhanpur, Bhopal \\ Professor People's College of Medical Sciences, Medicine People's College of Medical Sciences, \\ Bhanpur bypass road, Bhopal
}

\begin{abstract}
This is a report of a 50 year old lady with quadriparasis who was extensively evaluated before presenting to us with imaging and nerve conduction study and diagnosed as sensory motor predominantly axonal polyneuropathy of unexplained etiology. When we evaluate the patient and a chest X-ray and further work up revealed a surprise finding.

Keywords: Polyneuropathy, paraneoplastic syndrome, Small cell lung cancer
\end{abstract}

Accepted Date: 15 June 2013

\section{Case Report}

A 50 years old lady developed tingling, numbness and itching 11 months back in both her hands up to her elbows which progressed to both feet up to her knees. Her problem was acute in onset and progressive in nature with no aggravating factor and she felt slightly relieved on taking medication prescribed by her local health care worker. After few days patient developed weakness in all four limbs which was progressive and within 15 days patient was unable to do her routine activities, and had associated twitching of muscles in both upper limbs along with the development of significant muscular atrophy in all four limbs within 1 month. She consulted elsewhere before presenting to us and was advised -all routine investigations including, CT Scan head and cervical spine, which were within normal limits. Her nerve conduction study was suggestive of sensory motor predominantly axonal polyneuropathy affecting all four limbs. For this problem patient consulted various neurologists but could not get relief and her weakness and sensory symptoms progressed.

On questioning she also complained of left sided chest pain since 11months which was acute in onset, nonradiating, dull aching and intermittent in nature. It was associated with cough which was on and off for 2 month with mild expectoration.

On examination during her current admission with us, patient was found numbness tingling, weakness (Grade 1) and fasciculation's present in all four limbs with absent reflex. .On examination of her respiratory system patient was found to have absent breath sounds in left lower chest

Her Chest X ray (Figure 1) and CT chest (Figure 2,3) revealed the root cause of her gradual quadriparasis which had remained undetected till date due to previous focus on just the neurological manifestations of her actual disease.

The diagnosis was further confirmed on Bronchoscopy with biopsy (Figure 4) which showed. - A big mass lesion. On histopathology of biopsy shows malignant cells with nuclear moulding, minimam amount of cytoplasm, and stippled chromatin. S/o small cell variety of Bronchogenic carcinoma.

\section{Discussion}

In 1998 Massimo Camerlingo et al reported a study in which more than one third of the patients with peripheral sensory neuropathy of unexplained cause developed cancer . ${ }^{1}$ Small cell lung cancer is the most common cause of cancer-related death in men and women, and is responsible for 1.38 million deaths annually in developing country like India. ${ }^{2}$ It is highly lethal, with only a small minority of those diagnosed alive at 5 years. An interesting aspect of small cell lung cancer is the potential to produce distant clinical manifestations called paraneoplastic syndrome (7\% to $15 \%$ ). This syndrome most commonly presents as a peripheral sensory neuropathy, clinically observed in as many as $45 \%$ of patients, but "true" paraneoplastic neuromyopathy was reported in $7 \%$ patients. ${ }^{3}$ The concept of a remote or indirect effect of cancer on the nervous system arose in the first half of the twentieth ${ }^{4}$ and in the latter half of the twentieth century, the autoimmune etiology of many 
paraneoplastic disorders evolved, beginning with the discovery of antineuronal antibodies in patients with small cell lung cancer. ${ }^{3}$ On further studies the finding of anti-Hu antibodies can draw clinical attention but results that are negative for anti-Hu antibodies do not rule out small cell lung cancer. ${ }^{6}$ Therefore, there is a need for an appropriate clinical strategy for patients with peripheral sensory neuropathy of unexplained cause because it is very difficult to find out an occult neoplasm. So we have to evaluate such type of patients completely with paraneoplastic clinical expression in our mind. Diagnostic investigations should not limited to, complete blood count, chemistry panel, serum and urine protein and immunoelectrophoresis to detect monoclonal protein, a serological paraneoplastic autoantibody panel, nerve conduction studies/electromyography, mammography, chest X-ray, and CT or MR imaging of the chest, abdomen, and pelvis. In cases where occult malignancy is strongly suspected, serial testing should be necessary. ${ }^{5,7}$

\section{References}

[1]. Massimo Camerlingo, MD; Raffaello Nemni. Malignancy and Sensory Neuropathy of Unexplained Cause. Arch Neurol. 1998;55(7):981-984.

[2]. Thankappan KR \& Thresia CU.Tobacco use \& social status in Kerala.Indian J Med Res 2007;126:300-308

[3]. Croft PB,Wilkinson M. The incidence of carcinomatous neuromyopathy with special reference to lung carcinoma .In:Brain R and Norris FH eds. The remote effects of cancer on the nervous system . New York : Grune and Stratton,1965:44-54

[4]. Oppenhein H: Uber Hirnsymptome bei Carcinomatose ohne nachweisbare veranderungen im Gehirn.Charite-Annalen (Berlin) 1888;13:335-344

[5]. Richardson GE, Johnson BE (1992) Paraneoplastic syndromes in lung cancer. Curr Opin Oncol 4, 323-333.

[6]. Seute T, Leffers P, ten Velde GPM, Twijnstra A (2004) Neurologic disorders in consecutive patients with small cell lung carcinoma. Cancer 100, 801-806.

[7]. Rosenfeld MR, Dalmau J (2003) Current therapies for paraneoplastic neurologic syndromes. Curr Treat Options Neurol 5, 69-77.

\section{LEARNING POINTS-}

A symptom attributed to one system may have its origin in another major system involvement that may itself manifest later than its own complication.

We must do complete evaluation including $\mathrm{X}$ ray chest

Fig-1 X-ray chest
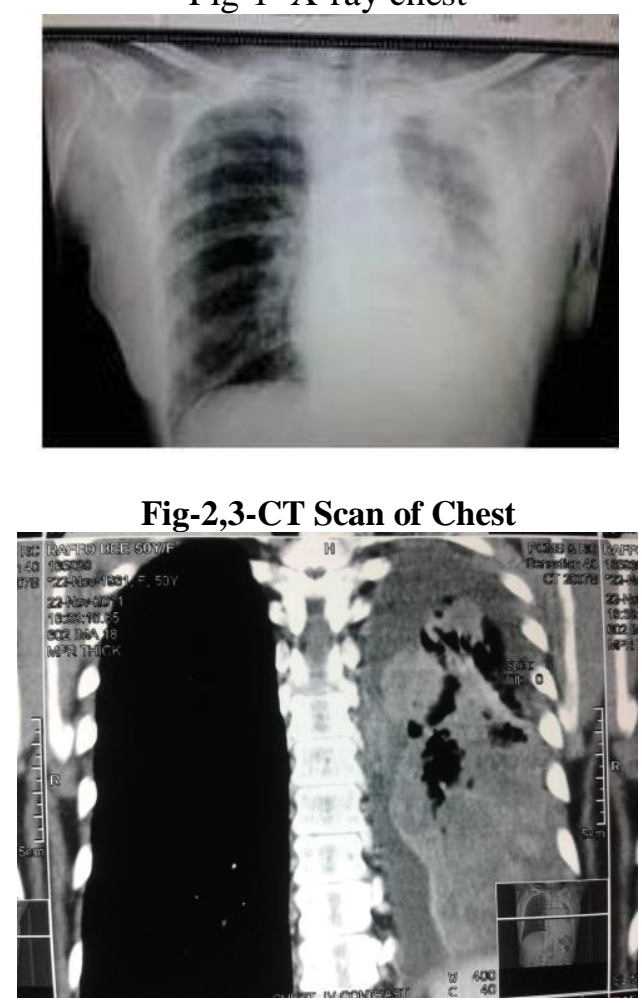


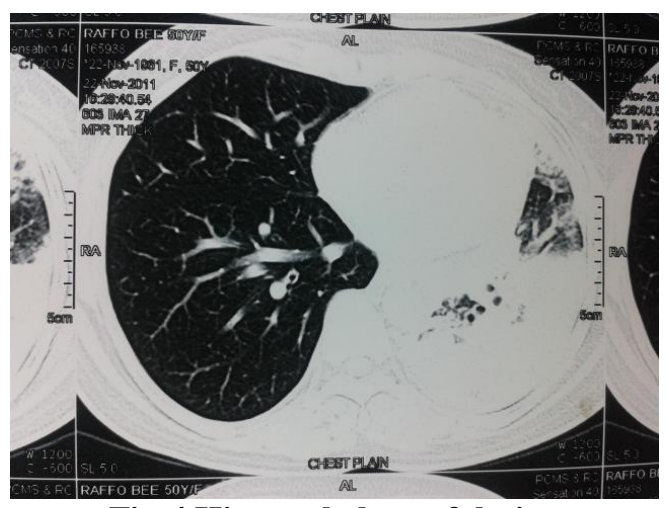

Fig-4 Histopathology of lesion

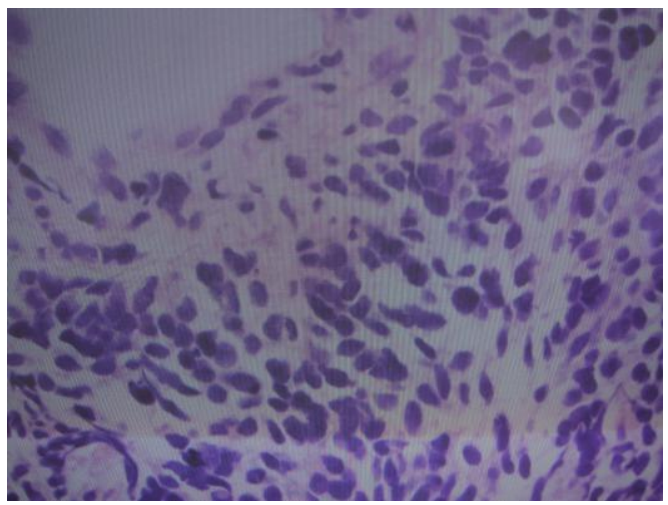

